\title{
Cytidine Analog RX-3117
}

National Cancer Institute

\section{Source}

National Cancer Institute. Cytidine Analog RX-3117. NCI Thesaurus. Code C113444.

An orally available small molecule and nucleoside antimetabolite with potential

antineoplastic activity. Upon administration, the cytidine analog RX-3117 is taken up by cells through a carrier-mediated transporter, phosphorylated by uridine cytidine kinase (UCK) and then further phosphorylated to its diphosphate (RX-DP) and triphosphate forms (RX-TP). The triphosphate form is incorporated into RNA and inhibits RNA synthesis. The diphosphate RX-DP is reduced by ribonucleotide reductase (RR) to dRXDP; its triphosphate form (dRX-TP) is incorporated into DNA. In addition, RX-3117 also inhibits DNA methyltransferase 1 (DNMT1). This eventually leads to cell cycle arrest and the induction of apoptosis. UCK is the rate-limiting enzyme in the pyrimidine-nucleotide salvage pathway. 